\title{
Lo filosófico: la obsesiva e ineludible tensión presencia-ausencia
}

The philosophical: the obsessive and unavoidable tension presence-absence

Marisa Berttolini*

Universidade del Uruguai

Resumen En el artículo se discute y problematiza la posibilidad del acontecimiento filosófico en las aulas de enseñanza media. Se reconoce el contexto desafiante en que se desarrolla la educación hoy, con una profunda diversidad de los educandos y un creciente malestar docente que atraviesa las instituciones, En este escenario se instalan frecuentemente profecías de fracaso. La reflexión intenta desmontar y refutar los argumentos más habituales con que se fundamenta la imposibilidad de desplegar el pensamiento filosófico en las aulas de adolescentes. Desde esa perspectiva se propone la deconstrucción crítica de una clase de filosofía como ocasión para repensar, desde las prácticas, las condiciones de posibilidad de la experiencia filosófica.

PALABRAS-ClaVE: Lo filosófico, Educación media, Discurso docente, Prácticas de aula.

Abstract This article discusses and problematizes the possibility of philosophy happening in the secondary school classroom. The article recognizes the challenging context in which education takes place, featuring a profound diversity of learners and a growing teacher malaise that crosses institutions.In this scenario prophesies of failure are often made. The reflection on this article tries to decompose and refute the most common arguments that support the idea that philosophical thinking in teenage classes is not possible. From this perspective, the article proposes the critical deconstruction of a philosophy class, which provides the opportunity to rethink the conditions in which philosophical experience is posible.

KEYWORDS: The philosophical, Secondary school, Teacher's discourse, Classroom practice. 
¡Si tienen la verdad, guárdenla! Lisboa revisitada Fernando Pessoa

Algunas cuestiones, que por su problematicidad atraviesan siempre los vínculos en la práctica docente que pretende ser filosófica pueden traducirse a estas preguntas: ¿Puede acontecer hoy el encuentro filosófico en las aulas de educación media? ¿Quiénes pueden ser interlocutores del diálogo filosófico?

Todos estamos involucrados, estudiantes de secundaria, profesores de didáctica, practicantes, docentes de filosofía, pues tienen que ver con el sentido de lo que hacemos. Son cuestiones que nos atormentan, que generan conflictos, que no están laudadas, sobre las cuales no hay consenso, que remiten a tensiones..., es decir que son filosóficas. Al mínimo análisis las preguntas se vuelven complejas y oscuras y se hace necesario desmontarlas.

Seguramente la recepción ingenua de las interrogantes nos impulsa sólo a sospechar de los estudiantes, perspectiva que consideramos reductiva. Creemos imprescindible preguntarse muy especialmente por la disposición de los docentes para generar un espacio filosófico. Y lo más importante, las dos cuestiones explicitadas remiten a la pregunta original, que al decir de Lyotard (1996) tiene necesidad de ser continuamente recordada, restablecida, porque se hunde, porque se nos escapa entre los dedos: ¿Cuál es la especificidad de lo filosófico? Los nuevos escenarios en que se despliega la educación, obligan a radicalizar las preguntas, quizás a reformularlas, a pensarlas desde otras miradas.

La Educación está siendo interpelada por una profunda y novedosa diversidad de sus educandos, frente a la cual sólo se exploran tímidas estrategias. Esta a diversidad puede comprenderse en clave de multiculturalidad (no necesariamente étnica, sino social, económica, cultural). Estos grupos constituyen ámbitos culturales alternativos, con una sensibilidad propia, con estrategias de supervivencia, con marcos axiológicos más o menos explícitos, con formas propias de resolver conflictos. Visualizamos que se están desarrollando procesos de construcción de nuevas formas de sociabilidad, de nuevas subjetividades irreductibles a categorías tradicionales. Hoy esa diversidad opera fundamentalmente como generadora de violencia, de prácticas de encapsulamiento fragmentado que tiene efectos significativos en el vínculo pedagógico. El deseo de aprender parece circular cada vez con menos fuerza en las aulas de adolescentes. Quizás persiste el deseo de saber, pero sin que lo acompañe la disposición para el esfuerzo que implica aprender. Es decir, se multiplican las resistencias y muchos alumnos no pueden o no quieren participar en el juego pedagógico. (BERTTOLINI, M, 2006).

El perfil de los docentes también ha cambiado. Desesperanza, malestar, multiempleo, nostalgia por los alumnos del pasado, desvaloración de la profesión, crisis de las instituciones. Reconocido el escenario educativo original y desafiante, es necesario tener en cuenta los discursos que parecen haberse instalado con más frecuencia, que se impregnan del fuerte malestar docente y que actúan como profecías 
de fracaso. Manifiestan un profundo pesimismo que ha ganado a muchos profesores y que se expresa a modo de letanía: no se puede, con estos estudiantes es imposible, no bay nada que bacer...

Merecen atención dos argumentos que tienen fuerza, dos objeciones habituales a la posibilidad de desplegar el pensamiento filosófico en las aulas de adolescentes. Se van a explicitar, para intentar desmontarlos y refutarlos.

Una objeción se refiere a lo técnico. Muchos colegas afirman: los estudiantes no tienen herramientas minimas, no manejan un vocabulario técnico, no pueden escribir, la oralidad es deficiente. Por lo tanto, no pueden filosofar.

El otro argumento pone en juego la cuestión del deseo, más específicamente de la ausencia de deseo. Se traduce, en la cotidianeidad, en las fórmulas reiteradas hasta el hartazgo: no quieren nada, están en otra, no les interesa pensar. El deseo parece estar anulado o más bien saturado por una cultura que indica siempre el objeto que se debe desear, que domestica el deseo, que lo transforma en voluntad de apropiación, de consumo, de dominación. Fuera de este circuito no se experimenta la falta.

Sobre la primera objeción, es bien discutible que el dominio de un vocabulario técnico sea condición para que acontezca lo filosófico. En todo caso, ¿qué vocabulario? Porque en filosofía no hay en sentido estricto un código general. Cada filósofo, cada sistema construye unas semántica específica, crea conceptos para que pueda irrumpir la novedad en el pensamiento. De esta manera, cualquier aprendiz de filósofo resulta analfabeto, incompetente, enfrentado a una doctrina que no conoce. Y peor, siempre está el riesgo de introducir, en base a preconceptos, ruidos en la comunicación con los textos. Por lo tanto, si el dominio a priori del vocabulario técnico fuera condición para participar como interlocutor legítimo en el diálogo filosófico, se inhabilitaría, aún para los filósofos, todo diálogo con la historia de la filosofía y aún con los contemporáneos. (ver REVEL,1962).

Si buceamos en la tradición del diálogo como soporte de la práctica filosófica, los criterios que a través de Platón, Sócrates pone como condición, tienen poco que ver con un vocabulario técnico. Se refieren más bien a actitudes, a disposiciones. En El primer Alcibiades (PLATON, 1871), el eje central es la preparación adecuada de Alcibíades para ejercer con justicia el arte de la política. El intercambio de Sócrates con su alumno es ocasión para destacar las condiciones rigurosas del diálogo filosóficamente fecundo, en tanto crítica punzante y sospecha atenta, pero que sólo puede sostenerse en un ámbito de profunda hospitalidad entre los protagonistas. A lo largo de la obra, Sócrates, con la intención de involucrar a Alcibíades en su propósito, va desplegando las exigencias: deseo de saber cómo piensa el otro, paciencia en la escucha, compromiso, reconocimiento de la propia ignorancia, persistencia en la búsqueda de la verdad, dedicación del tiempo propicio para la reflexión en profundidad, evitando la ansiedad por encontrar respuestas concluyentes, valor para expresar el propio pensamiento, habilidad para sopesar la oportunidad de las intervenciones, disposición al perfeccionamiento, a conocerse a sí mismo y al cuidado de sí. 
A su vez, Sócrates denuncia los obstáculos: la autosuficiencia de quien siente que no necesita de nadie, la complacencia en las propias "perfecciones", la vanidad, y desprecio por los atributos del interlocutor, la vana aspiración a la riqueza y al poder, como voluntad de dominio sobre los otros hombres.

Las condiciones poco tienen que ver con el dominio de una técnica intelectual o discursiva. Después de todo, una técnica en sentido instrumental no es garantía en sí misma de buen pensamiento. Sirve para pensar como para no pensar, para identificar y tratar creativamente problemas fermentales, como para eludirlos. Los requisitos socráticos son, en cambio, verdaderos preceptos éticos vinculados a la sabiduría, al cuidado de sí y de la polis.

Entonces la lengua del iniciado, del profano en filosofía, no debería ser un obstáculo, sino la oportunidad de instalar la comunicación y el proceso de interpelación de la práctica filosófica.

Por otra parte, una técnica en sí misma no es garantía, porque es neutra en relación a nuestro problema. El vocabulario propio de un sistema puede constituirse en una verdadera prisión mental que obstaculiza el pensamiento innovador.

En este sentido el filósofo uruguayo Carlos Vaz Ferreira, en su obra Lógica Viva (1962) propone el "pensar por ideas a tener en cuenta" como alternativa al "pensar por sistemas". La distinción tiene en perspectiva la polémica con el positvismo, que aparecía como sistema hegemónico en su época, pero trasciende esa coyuntura y supone la defensa de un modo de hacer Filosofía. Vaz Ferreira desconfía de los sistemas filosóficos por su carácter de totalidad cerrada, de esquematización que lleva incluso a forzar la realidad para encuadrarla en la red conceptual. En cambio, "el pensar por ideas a tener en cuenta" abre la reflexión, facilita una mejor comprensión del mundo en toda su complejidad, siempre resistente a dejarse atrapar en prisiones conceptuales. Es un modo de pensar que exige constante originalidad y rigurosidad, que tensiona porque inhabilita toda respuesta programada, todo dogmatismo. Se trata de un modo de escepticismo que puede identificarse con la Filosofía misma. Es casi una exigencia ética para el filósofo aplicar su mirada lúcida, haciendo uso de la duda orientada a destruir y reconstruir la argumentación. La seguridad, la comodidad intelectual no son compañeras de la Filosofía. La conciencia filosófica es corrosiva, vive en desasosiego, en conflicto consigo misma. Vaz Ferreira sustenta una razonabilidad que se aparta tanto de la racionalidad instrumental como de la racionalidad omnicomprensiva y totalizante. Es un pensamiento que asume la incertidumbre y aún más, se regocija en ella.

Pasemos al otro argumento. Si aceptamos que el deseo es condición necesaria para que ocurra el acontecimiento educativo, el problema se desplaza. Si no aparece espontáneamente, ¿podemos enseñarlo? La cuestión del deseo y la posibilidad de enseñar o no el amor por la filosofía, ha sido objeto de reflexión entre colegas que insisten en pensar filosóficamente la educación filosófica. Y las conclusiones muestran matices: Alejandro Cerletti (2007) sostiene: "El deseo, como el deseo de pensar, es, en última instancia intransmisible, no es posible enseñar a amar la sabiduría, como por cierto no es posible enseñar a enamorarse." Por su parte, Olga Grau (2009) reflexiona: "Al parecer lo inducimos, lo producimos, hacemos deseable el deseo de pensar, enseña- 
mos el deseo de pensar(...) Enseñar el deseo por la filosofía es mostrar al otro nuestro propio deseo, exponernos en nuestra propia vacilación, indicar el movimiento de la incertidumbre."

Por su parte, en una entrevista en el marco de una investigación, un profesor arriesga:

"Yo creo más en la seducción y en la posibilidad a partir de un discurso, de establecer una estrategia de inculcar enigmas en los estudiantes....No sé bien lo que es una clase con rigor filosófico. A mi me pasa por ejemplo que hace tiempo abandoné a los presocráticos. $Y$ volver a dar los presocráticos me obligó a releer un montón de cosas. $Y$ en esa relectura a partir de la preparación de clases empecé a revisar un montón de conceptos que yo tenía de los presocráticos y que los empecé a modificar. Yo creo que esa continua revisión o esa relectura es lo que le debería de dar cierta solidez, por lo menos académica, a lo que doy."

El docente apuesta a la seducción para provocar el deseo, el deseo de filosofar, de arriesgarse al juego intelectual. Pero la seducción a la que apela el docente, no es la banalización y el simulacro. El profesor relata su propia aventura filosófica. Fue capaz de releer, desde otros lugares. Descubre, modifica, resignifica su diálogo con la historia de la filosofía. Hace un movimiento que socava la indiferencia y la rutinización. En una palabra: piensa. Y es esto lo que se puede "mostrar" y así producir la seducción filosóficamente fecunda. La práctica pedagógica no queda reducida a la circulación de saberes. Se provocan cambios en la experiencia de docentes y educandos, se produce un trabajo esencialmente filosófico de transformación de las subjetividades. $\mathrm{La}$ tarea no es entonces sustituir un saber por otro, saturando el deseo. Se trata en cambio de trastocar la relación habitual con el saber, alimentando el deseo a través de la transformación de la experiencia de sí. El docente, sólo puede inducir el proceso si él ha transitado esta práctica, sólo si cuenta con estas experiencias, podrá ponerlas a disposición.

Pero reconozcamos, la estrategia de la seducción, como buena apuesta no va acompañada de la certeza, los efectos posibles son contingentes, y a largo plazo, quizás se concreten mucho después de que concluya esta relación pedagógica.

\section{Las prácticas en la Educación Filosófica: deconstrucción filosófica}

El centro de la reflexión es la deconstrucción crítica de una clase de Filosofía presentada por una practicante, alumna de un curso de Didáctica de Filosofía, en el Instituto de Profesores Artigas de Uruguay. ${ }^{1}$

El análisis de clases es una actividad relevante en los cursos de Didáctica en el sentido de permitir que se pongan en evidencia todos los significados posibles de la práctica.

Las representaciones sobre la Didáctica, sobre las posibilidades de la educación filosófica, sobre la exigencia de un abordaje filosófico y político de la enseñanza de la Filosofía son el horizonte que orienta la intencionalidad del análisis. 


\section{Relato de la clase sobre notas y reconstrucción de la memoria}

Un liceo céntrico de Montevideo. 1er año de Educación media superior.

Asistentes: 25 alumnos entre 15 y 17 años, profesora del curso, practicante, profesora de didáctica, otra practicante.

Asignatura Filosofía. Carga horaria, 3 horas semanales ${ }^{2}$

Los alumnos están distribuidos espacialmente en la disposición tradicional. Filas de bancos, todos orientados hacia el pizarrón y el escritorio del docente. Los observadores nos ubicamos al fondo del salón. La clase va a tener una duración de 40 minutos. Al comienzo los estudiantes necesitan cierto tiempo de acomodación, llegan excitados del recreo, se van instalando, comentan sobre la presencia de los observadores,... El pasaje de la lista funciona como regulador para los estudiantes y reduce la ansiedad de la practicante. Poco a poco se dan las condiciones de posibilidad para el vínculo pedagógico. El salón tiene mala acústica y resuenan los ruidos del corredor permanentemente. Pero esto no parece perturbar a nadie (salvo a mí, claro), pues seguramente es el habitual telón de fondo en que se trabaja.

Practicante: (Pasa la lista)

P: Les había dejado un texto en la fotocopiadora, Lo trajeron? No sé si lo leyeron. (Sólo algunos alumnos lo tenían, y entonces reparte algunas copias del texto) Vamos a trabajar sobre un texto del filósofo Parménides de Elea, del siglo VI A.C. ¿Qué les dice el siglo VI A.C.?

A1.1: La ciudad- estado, el surgimiento de la moneda.

P: ¿Qué significa la moneda?

Al. 2: Está vinculado con la capacidad de abstracción, con la racionalidad, con el comienzo de la Filosofía.

P: ¿Y cuál es la función de la Filosofía?

Al .3: Problematizar la realidad.

P: Vamos a pasar a leer el texto de Parménides, Primero vamos a hacer una lectura completa del fragmento que seleccioné, en voz alta. Voluntarios? (se ofrecen 3 varones y lee cada uno una parte).

P: ¿Qué entendieron?, ¿Qué sintieron?

Al. 3: Es la historia de un joven que lo transportan hacia una Diosa que le va a decir la verdad.

P: ¿Otra opinión? Silencio ¿Cuál es la forma del texto?

A1. 4: Es un Poema

Ala.5: Yo me sentí decepcionada, porque sabíamos que los filósofos abandonaban los Dioses y lo primero que dice es que la verdad se la va a mostrar la Diosa.

Ala. 6: Pero no hay un crac total, sobreviven elementos del mito.

Al. 3: Los cambios se dan en un proceso. 
P: El texto, ¿̇les pareció sencillo?

Als: Siiiii. (en coro)

P: Vamos a realizar ahora un trabajo más analítico, vamos a profundizar el abordaje del texto. ¿De qué habla el texto? ¿ Qué es lo que plantea?

Al. 7: Hay un camino, una luz,

$\mathrm{N}$ : Intenten un análisis literario. ¿Qué significan la noche y el día?

Als varios: La noche representa la mentira, la ignorancia, el no saber, lo falso. El día, la verdad, el saber.

P: La vía del conocimiento se denomina "episteme". La noche es la vía de la opinión, la "doxa”. Ustedes, ¿cuál elegirían?

Ala. 5: Bueno, si me hubieran criado de otra manera, si mi madre me hubiera mostrado lo bueno como malo y a la inversa, elegiría diferente. Voy a elegir según lo que me hayan enseñado.

Al. 3: La mayoría opta por el camino de la noche, sólo un grupo de sabios, elige el día.

Al. 8: Yo, si fuera por mí, preferiría la noche...., pero sé, que se debe elegir el día.

Ala. 6: Para mí, el joven recorrió primero el otro camino, y sólo más tarde elige el día. Y eso pasa con todos.

(Timbre)

P: Lean más cuidadosamente el texto para la clase que viene y seguimos interpretándolo

\section{Deconstrucción y reconstrucción crítica ${ }^{3}$ (o de los modos en que los alumnos dejan perplejos a sus docentes,.....porque los docentes se dejan conmover).}

La reflexión se focaliza especialmente en la formulación de preguntas. Y esto en dos sentidos: la pregunta como recurso didáctico para organizar los contenidos, promover la participación de los estudiantes y construir colectivamente el conocimiento, y la pregunta inquietante, como traducción de un problema reconocido, para invitar a la indagación filosófica.

Las primeras preguntas que instala la practicante.: iQué les dice el siglo VI A.C.? ¿̇Qué significa la moneda?, tienen por objetivo una revisión de la información que ha circulado antes en el curso, como marco para instalar el texto de Parménides. La practicante renuncia acertadamente a aportar expositivamente los datos y apuesta mediante preguntas a que la síntesis la hagan los alumnos, procedimiento que significa además una instancia de evaluación en proceso. Sin embargo, la modalidad de las preguntas habilita la reproducción mecánica de contenidos complejos, cuya apropiación ha requerido seguramente procesos pedagógicos y filosóficos previos. Al ser repetidos por los alumnos como conclusiones evidentes, se debilita el valor provocativo de los enunciados y circulan como malas caricaturas del pensamiento filosófico. Me parece que el ejemplo más notorio es cuando ante la pregunta ¿Cuál es la función de la filosofía?, 
un alumno responde: problematizar la realidad, (seguro de que esa es la intervención esperada y "filosóficamente correcta"). La fórmula enunciada desde la certeza y a modo de consigna, resuena como expresión dogmática y paradojalmente antifilosófica.

Las preguntas: ¿¿ué entendieron? ¿Qué sintieron?, que se introducen para generar las primeras aproximaciones al texto son pedagógicamente ambivalentes: son buenas preguntas porque su amplitud habilita intervenciones variadas, invitan a participar desde distintos niveles de comprensión y evitan la inhibición de aquel estudiante que siente que muchas cosas aún le resultan oscuras. Esa misma cualidad, el carácter tan abierto de las preguntas, puede desconcertar a los alumnos sobre su verdadera intención y bloquear su participación.

En la instancia de devolución, la pregunta ¿qué sintieron? es fundamentada por la practicante en este sentido: "la finalidad es que todos los alumnos se sientan interpelados. Pueden no haber comprendido el texto, pero no es posible que el texto no provoque su sensibilidad, al menos generando la perplejidad intelectual frente a un mensaje oscuro y poco inteligible".

En el orden de las representaciones previas a modo de "horizonte de expectativa" de la practicante, predominaba la idea de que los alumnos iban a manifestar desorientación frente al mensaje y que su intervención como docente debía orientarse a apoyar el nivel de comprensión lectora y decodificación semántica, mucho antes de que pudiera emprenderse cualquier intento de interpretación crítico- filosófica. Así lo había manifestado y lo habíamos trabajado en la etapa de planificación de la clase.

Los aportes de algunos alumnos le imprimieron un giro muy distinto al proceso de reflexión, cuestión que funcionó como un obstáculo pedagógico fuerte para la practicante. Tuvo dificultades para tomar decisiones que no estaban previstas y para potenciar las observaciones inteligentes y muy pertinentes de algunos estudiantes.

La alumna 5 responde: Yo me sentí decepcionada, porque sabiamos que los filósofos abandonaban los Dioses y lo primero que dice es que la verdad se la va a mostrar una Diosa. Si bien la intervención se mantiene en el plano en que se había interpelado, pues frente a la pregunta, ¿̇qué sintieron?, la estudiante afirma: Yo me sentí decepcionada, la respuesta se ubica en un registro muy diferente al esperado. Hay varias habilidades intelectuales puestas en juego, comprensión, capacidad de relacionar, identificación de un aparente conflicto con ideas previas, el reconocimiento de un problema. La alumna instala una interesante cuestión a investigar. La respuesta lejos de cerrar definitivamente la cuestión, abre un nivel de insatisfacción cuyo sentido es el deseo de saber, imprescindible en el vínculo pedagógico, pero que la practicante no aprovecha. Son otros compañeros lo que marcan el matiz: Pero no hay un crac total, sobreviven los mitos. Los cambios se dan en un proceso. Sería una buena oportunidad para profundizar el análisis del Poema y mostrar la diferencia sutil pero relevante entre referirse a la Diosa de la verdad y la Diosa Verdad. O de otra forma, a la Verdad Divinizada.

Luego se emprende un trabajo analítico sobre el texto y se seleccionan las imágenes de la noche y el día en su valor metafórico. Rápidamente los alumnos decodifican sus posibles sentidos: La noche representa la mentira, la ignorancia, el no saber, lo 
falso. El día, la verdad, el saber. Resuena el eco de la tradición cultural occidental y su trillada simbología.

La practicante introduce los términos técnicos "doxa” y "episteme" y los explica. A continuación pregunta: $U d s$, i̇cuál elegirían? Aparentemente la pregunta convoca la autonomía del estudiante, abre una opción que el alumno podrá hacer libremente y que tendrá que fundamentar, es decir, la pregunta parece estimular la experiencia intelectual de su destinatario. Pero desde otra perspectiva es muy poco fermental. Encierra supuestos maniqueos. Fácilmente puede perderse su valor de indagación para transformarse en una pregunta retórica, la disyunción puede disolverse y la interrogante sugiere sólo una respuesta gnoseológica y éticamente "correcta". Es decir, sólo se puede y se "debe" elegir la imagen del día, la "episteme".

Pero la alumna 5 desplaza el eje de la reflexión. No contesta lo que se preguntó, da un salto cualitativo y propone un metanálisis, explicitando los motivos por los que solución parece obvia: Bueno, si me hubieran criado de otra manera, si mi madre me hubiera mostrado lo bueno como malo y a la inversa, elegiría diferente. Voy a elegir según lo que me hayan enseñado. El eje de la reflexión se desplaza. Lo obvio se vuelve problema. La intervención remite a supuestos nítidamente filosóficos y fuertemente polémicos como el relativismo cultural de los valores éticos y la función reproductora de la educación. Incluso, en el discurso se filtra una cuestión de género, es a la madre a quien se responsabiliza como transmisora de valores.

Se suceden las intervenciones. Unos contestan a otros. Hay escucha y capacidad de diálogo.

En el mismo registro anterior, pero con otra perspectiva un alumno acota: Yo, si fuera por mí elegiría la noche...pero sé que se debe elegir el día. Logra ejemplificar el conflicto ético por excelencia: la tensión entre lo que queremos y lo que debemos. Y remitiéndonos a la tradición filosófica podríamos preguntarnos, ¿lo sé por intuición, lo sé como imperativo que emana de la razón práctica, lo sé porque forma parte de la ideología hegemónica en mi sociedad?

\section{¿Fue una buena clase de Filosofía? ¿Circuló la experiencia filosófica?}

Sin duda no hubo una relación causal mecánica entre las intervenciones de la practicante y el acontecimiento pedagógico que ocurrió. Pero, ¿hay un vínculo causal entre lo que se enseña y lo que se aprende? Una clase, ¿no es siempre un encuentro imprevisible? Parece claro que la propuesta pedagógica habilitó (o al menos no bloqueó) el pensamiento crítico-filosófico, la construcción colectiva del conocimiento y el reconocimiento de núcleos problemáticos.

En el aula, las redes de poder que atraviesan el vínculo pedagógico, se desestructuran y se reorganizan de otras maneras. Por momentos el poder se concentra en la docente que es quien monopoliza la pregunta y por lo tanto direcciona la construcción del conocimiento. Pero los aportes de los alumnos modifican la estructura inicial y el poder - saber circula en varias direcciones, se democratiza, se comparte. 
Según la practicante y la profesora adscriptora, el estilo de participación de la clase es habitualmente diferente. Participan muchos alumnos y con cierto grado de ansiedad que provoca desorden y dificultad para la escucha. En la clase visitada el ritmo cambió. (Habitualmente los alumnos cuidan a los practicantes, ante la presencia invasiva de los observadores, administrando sus intervenciones) Los aportes recayeron en pocos estudiantes, pero fueron de alta calidad. Se identifican algunos perfiles típicos: la alumna 5 intervino desde la problematización, instaló la transgresión de las reglas de juego previstas y generó una dosis de perplejidad en todos los actores (y observadores). La alumna 6 funcionó como la académica, introdujo el matiz, la precisión, la síntesis. Así, la creatividad y el rigor circularon amalgamados y la clase se construyó como un espacio de valor filosófico.

El acontecimiento pedagógico resulta paradojal. La intencionalidad que lo atraviesa, y que se explicita en la planificación de la practicante, es provocar una enseñanza transformadora, pero se traduce en un formato mimético, que fundamentalmente requiere la reproducción de los saberes aprendidos.

La debilidad mayor de la propuesta tuvo que ver con la dificultad para identificar y potenciar la sensibilidad filosófica en las observaciones de algunos estudiantes. Pero he aquí la paradoja interesante: la inexperiencia, vivenciada con cierta dosis de impotencia de la docente (que no logra poner la pregunta fermental ni trabajar filosóficamente las intervenciones), hace que se repliegue y que no se posicione desde el saber y la destreza en la conducción pedagógica. Pero, justamente, esta modalidad de administrar su rol, desde el no saber o no poder, permite que los alumnos tomen la palabra, viabiliza el ejercicio del poder intelectual del otro, que aflore la capacidad de descubrimiento y la creatividad para proponer. Muchas veces el docente experiente, aun contra su voluntad, obtura este proceso y queda atrapado por la propia solvencia y la ansiedad por hacer circular su saber.

¿Cómo interpretar la paradoja? ¿Quizás en el registro del polémico maestro ignorante de Rancière, que invierte la lógica del maestro explicador?

La explicación no es necesaria para socorrer una incapacidad de comprender. Es, por el contrario, esa incapacidad, la ficción estructurante de la concepción explicadora del mundo. Es el explicador que tiene necesidad del incapaz, y no al contrario, es él que constituye al incapaz como tal. Explicar alguna cosa a alguien es, antes que nada, demostrarle que no puede comprender por sí mismo. Antes de ser un acto de pedagogo, la explicación es el mito, la parábola de un mundo dividido en espíritus sabios y espíritus ignorantes. (RANCIÈRE, J. 2002).

En la clase visitada se quebró la lógica de la explicación. La docente no explicó, pero también los alumnos resistieron la explicación.

Rancière opone al maestro explicador la imagen del maestro emancipador.

Puede enseñarse lo que se ignora, desde que se emancipa al alumno: esto es que se fuerza al alumno a usar su propia inteligencia. El ma- 
estro es aquel que encierra una inteligencia en un círculo arbitrario del cual no podrá salir si no se vuelve útil a sí misma..... El ignorante aprenderá solo lo que el maestro ignora, si el maestro cree que él puede, y lo obliga a actualizar su capacidad.

La tesis de Rancière resulta seductora pero discutible. Hay otra clave de interpretación. La intervención de la practicante se inserta en un curso donde el grupo ya ha hecho un largo proceso de iniciación filosófica con su profesora. El valor fermental que tuvo este encuentro tiene que ver también con el trabajo potente en lo filosófico y lo pedagógico que ha plasmado la docente adscriptora. Ha enseñado a ejercitar el pensamiento crítico, ha hecho circular sus saberes no para obtener la repetición de lo mismo, sino para ser recrearlos, para ser usados como cajas de herramientas para plasmar el pensamiento propio, para hacer la propia experiencia. Quizás no sea posible enseñar a pensar, y cualquiera sea la estrategia nunca hay garantías, pero atreverse a pensar con otros, es un buen comienzo.

\section{Referencias}

BERTTOLINI, M. La Educación filosófica para la reconstrucción de las subjetividades. en Kohan,W. (comp). Teoría y Práctica en filosofía con niños y jóvenes. Buenos Aires: Novedades Educativas. 2006

CERLETTI, A. La enseñanza de la filosofía: de la pregunta filosófica a la propuesta metodológica. Intervención en el II Encuentro Internacional de Filosofía y Educación. Universidade do Estado do Rio de Janeiro, 2007.

FOUCAULT, M. El otro como mediador. Hermenéutica del sujeto. (1994) Ediciones de la Piqueta. Madrid: Tercera Lección, 1982.

GRAU DUHART. O. Otra vez el deseo. Para pensar la enseñanza de la Filosofía. Revista de Filosofía, v. 65, 2009, p. 97-103.

LYOTARD, J. F. Memorial a propósito del curso filosófico, en La posmodernidad (explicada a los niños). Barcelona: Gedisa, 1996.

PLATÓN. El primer Alcibíades o De la naturaleza humana. Obras completas, edición de Patricio de Azcárate. Tomo 1, Madrid: 1871.

RANCIÈRE J. O mestre ignorante. Belo Horizonte: Autèntica Editora , 2002.

REVEL, J. F. ¿Para qué filósofos? Traducción de Juan Nuño. Escuela de Filosofía ARCIS. Caracas: Colección Avances 1, 1962. Edición electrónica www.philosophia.cl.

VAZ FERREIRA, C. Lógica viva. Buenos Aires: Losada, 1962

\section{Notas}

${ }^{1}$ En el Uruguay, la formación de docentes para la Educación Media, se desarrolla durante 4 años. En el currículo se incluyen asignaturas específicas de la disciplina y otras de formación pedagógica. El eje articulador esencial es el espacio de Didáctica, que comprende instancias teóricas con el docente especializado y una instancia práctica a lo largo de 3 años. Durante 2 años el estudiante participa, durante todo el curso, como observador en una clase de otro profesor adscriptor que funciona como "tutor" y donde hace algunas intervenciones y en el último año de su formación cada estudiante tiene un curso a su cargo con la supervisión del profesor de didáctica. 


\section{Marisa Berttolini}

${ }^{2}$ La asignatura Filosofía está incluida en los 3 años de Bachillerato en todas las orientaciones, con una carga horaria de 3 horas semanales.

${ }^{3}$ El nivel de la reconstrucción no se corresponde con los criterios de evaluación Se trata de una alumna de profesorado que está haciendo su experiencia de $1^{\text {a }}$ práctica y que ha dado sólo 2 y 3 clases antes de la visita. Por lo tanto es claro que hay muchos aspectos de contenido disciplinar y didáctico que aun no puede dominar. En las devoluciones, siempre colectivas, se profundiza el análisis y todos hacen observaciones exigentes, porque se consideran instancias fundamentales de aprendizaje.

* Docente de Didáctica de la Filosofía. Instituto de Profesores Artigas. Docente de Epistemología, CLAEH. Maestría en Docencia de la Educación Media. Montevideo, Uruguay.

\section{Correspondência}

Marisa Berttolini - Canelones 1929 apto 103, Montevideo, Uruguay. Código postal 11200.

E-mail:majal@adinet.com.uy

Recebido em 27 de outubro de 2014

Aprovado em 03 dezembro de 2014 